\title{
Guest editorial note
}

\author{
Andrea Trianni • Patrik Thollander
}

Published online: 21 April 2018

(C) Springer Science+Business Media B.V., part of Springer Nature 2018

Globally, industry is one of the largest energy end-using sectors, and thus also one of the key sectors in regard to climate change mitigation. Improved industrial energy efficiency in industry is a growing area of research ranging from pure production in companies, e.g. nanotechnology and additive manufacturing (AM), to how industrial energy systems can be optimised, up to national and international levels studying energy policy programme and its impact. In this special issue, a number of these areas are covered. Most likely, improved energy efficiency in regard to AM and digitalization are areas where key initiatives are now taking place. Also, policies for improvements and how digitalization can support and speed-up the improvement process are also areas of growing interest.

The first article by Aden in this Special Issue interestingly discusses the role of industrial energy efficiency in light of the global 2-degree pathway established in the 2015 Paris Agreement. The article provides an overview

This special issue has been produced in cooperation with the European Council for an Energy Efficient Economy.

\footnotetext{
A. Trianni $(\bowtie)$

Faculty of Engineering and IT, School of Systems, Management and Leadership, University of Technology Sydney, 81 Broadway, Ultimo, NSW 2007, Australia

e-mail: andrea.trianni@uts.edu.au

P. Thollander

Division of Energy Systems, Department of Management and Engineering, Linköping University, SE-581 83 Linköping, Sweden

e-mail: patrik.thollander@liu.se
}

of the metrics of industry globally and in the USA, as well as industrial sector pathways and programmes for limiting average warming to $2^{\circ}$ this century. The paper also "assesses options for reducing industrial sector GHG emissions via voluntary programs and sectoral approaches".

As climate change concerns are strongly linked to other economic issues, it is worth considering the evolution of the industrial structure, considering more conventional technologies as well as new emerging ones. For what concerns the first, the discussion is brought by Xylia et al. for the steel industry, who offer "valuable insights on scrap availability and capacity development at the regional level for producers contemplating new investments". In the paper, findings from the application of a steel optimization model show that "regional availability, quality and trade patterns of scrap will influence production route choices, possibly in favour of secondary routes. Also policy instruments such as carbon taxation may affect investment choices, and favour more energy efficient and less carbon-intensive emerging technologies".

Regarding new manufacturing technologies, Hettesheimer et al. address the issue of quantifying the impact of additive manufacturing processes on energy demand by examining selective laser sintering (SLS). To this end, the authors "suggest and apply a model that focuses on three important phases in the lifecycle of additively manufactured components and which allows us to compare them to conventional manufacturing processes.", taking Germany as showcase, finding that 
"despite replacing only a small component, substantial energy savings can be achieved".

Further valuable savings at the national level could be achieved by looking at opportunities offered by industrial activities with effective energy surplus. This is the focus of the paper authored by Feta et al., where they evaluate the demand response (DR) potential that can be provided to the Dutch national grid by the integrated steel-making site of a large corporation. Results show that the company can effectively supply a relevant amount of power, with significant availability rate. Despite "this is not sufficient for participating in the current emergency capacity programs in the Netherlands, which require at least $20 \mathrm{MW}$ for longer than one programme time unit", the company "can provide a significant amount of power to the national grid".

Useful insights for policy makers could also come from a better understanding of the effective opportunities for improved efficiency in the use of heat. For this purpose, Rehfeldt et al. present a methodology to disaggregate Eurostat's energy balance for the industrial sector. Their results show that, "although a similar distribution of energy use by temperature level can be observed, there are considerable differences among individual countries", calling from differentiated actions from policy makers. Indeed, such "differences are mainly caused by the countries' heterogeneous economic structures, highlighting that approaches on a process level yield more differentiated results than those based on subsectors only".

Regarding a more efficient use of heat, an interesting discussion in another energy-intensive industry (glass industry) is brought in the paper authored by Karellas et al., where "a vertical approach" is utilised "to provide information on both ETS market evolution and specific technical information to support technological innovation to the glass industry". Authors have shown the results of a case study for a container glass furnace based on simulation results, where the impact of different operating and design configurations on specific energy consumption and $\mathrm{CO}_{2}$ emissions is investigated.

Despite the common belief that heat recovery is feasible and valuable almost exclusively for very high temperature processes, Schumm et al. present the "evaluation of functionality and potential of a hybrid heating system $\left(\mathrm{H}^{2} \mathrm{~S}\right)$ prototype", for low temperature applications, as applied in the food industry. The system increases "the energy efficiency and flexibility by integrating low temperature heat and responding to sudden changes in the demand and supply structure, like DR strategies on intermittent renewable energies and the changing availability of HW and steam". As authors note, the $\mathrm{H}^{2} \mathrm{~S}$ represents a "feasible solution for maintaining product quality and safety while also increasing energy efficiency and energy flexibility".

Energy efficiency does not necessarily mean new energy-efficient technologies, rather smarter ways of managing the existing set of installed ones, by an increase in the overall awareness of (industrial) final users. Enhanced knowledge and awareness can be reached in multiple ways, e.g. by supplying decision-makers with energy-saving advice based on smart metre data, as developed by Kimura et al. In that paper, the authors "developed a novel tool that, based on smart meter data, automatically generates customised energy-saving advice to commercial and industrial customers". In particular, the tool contains several approaches useful for a more aware energy-related decision, "such as fault detection, energy disaggregation, social comparison and benchmarking, and selective visualisation".

To the same side is the research by Mahapatra et al., where a deeper look to the behavioural change issues leading to energy efficiency in a manufacturing plant is considered. In their case study, the authors have tried to further understand the barriers and opportunities to influence behaviour of production workers and the corresponding energy savings potentials. Results based on interviews with actors involved in the energy efficiency project showed "that a concrete goal, the employment of a fulltime operational leader who earlier worked as a production worker, and the involvement of both the leadership and employees in project management represent keys to the success of the project".

Among others, increased awareness, change decision routines, and trust represent indeed critical key factors for the success of energy efficiency networks, as analysed by Dütschke et al. Moreover, authors remarked the importance of energy audits to make profitable potentials visible, as well as reduction in transaction costs.

Those factors could lead to an increased number of implemented EEMs, in both core processes and ancillary services, e.g. as shown by Nehler et al. pertaining compressed air systems in Swedish companies. Relying on previous literature insights, authors have investigated whether "technology-specific measures might encounter different barriers to and drivers for energy efficiency". Moreover, the authors have investigated whether "the same applies to the non-energy benefits 
(NEBs) related to energy efficiency improvement measures; since measures vary between various energyusing processes, the perceived NEBs might be different as well". Interestingly, "the results showed that the major barriers are related to the investment, or are of an organisational character, and that organisational and economic factors seemed to be important for making positive decisions on energy efficiency investments and measures in CASs. Major NEBs for CASs include productivity gains and the avoidance of capital expenditures."

This calls for policy-making actions to be more specific, better tailoring different target groups in industry, looking at "companies' characteristics and barriers to draw conclusions on energy efficiency policies and specific recommendations on energy efficiency measures.", as found by Wohlfarth et al. More in detail, thanks to their broad empirical investigation within German industries, the authors noted several differences in the implementation of EEMs according to, e.g. firm size, as well as different barriers hindering their adoption. Therefore, to support the implementation of EEMs by policies, a deeper understanding of the barriers affecting different kinds of companies is necessary.

Nevertheless, the implementation of energy efficiency measures could involve several decision-makers within a company. Therefore, as investigated by Cagno et al. within Italian manufacturing companies, it may be really important to also look at those decision-makers, who may "perceive different barriers about the same EEM. EEMs may be negatively affected by reasons related to other areas of industrial sustainability, whilst positive reciprocal impacts may exist among areas of industrial sustainability; thus, EEMs may have effects on areas other than energy efficiency, and these effects may be perceived only by such areas". Therefore, as the authors conclude, "results show that an industrial sustainability perspective can better explain the real decision-making process of adopting an EEM". 